\title{
Review Article \\ Which Patients Need an Axillary Clearance after Sentinel Node Biopsy?
}

\author{
Anastasia Pazaiti and Ian S. Fentiman \\ Research Oncology, Guy's Hospital, 3rd Floor Bermondsey Wing, London SE1 9RT, UK \\ Correspondence should be addressed to Ian S. Fentiman, ian.fentiman@gstt.nhs.uk \\ Received 4 April 2011; Revised 16 June 2011; Accepted 2 July 2011 \\ Academic Editor: Christoph Klein
}

Copyright () 2011 A. Pazaiti and I. S. Fentiman. This is an open access article distributed under the Creative Commons Attribution License, which permits unrestricted use, distribution, and reproduction in any medium, provided the original work is properly cited.

\begin{abstract}
Sentinel lymph node biopsy (SLNB) is a safe and accurate minimally invasive method for detecting axillary lymph node (ALN) involvement in the clinically negative axilla thereby reducing morbidity in patients who avoid unnecessary axillary lymph node dissection (ALND). Although current guidelines recommend completion ALND when macro- and micrometastatic diseases are identified by SLNB, the benefit of this surgical intervention is under debate. Additionally, the management of the axilla in the presence of isolated tumour cells (ITCs) in SLNB is questioned. Particularly controversial is the prognostic significance of minimal SLNB metastasis in relation to local recurrence and overall survival. Preliminary results of the recently published Z0011 trial suggest similar outcomes after SNB or ALND when the SN is positive, but this finding has to be interpreted with caution.
\end{abstract}

\section{Introduction}

For patients with operable breast cancer, the major prognostic determinant is whether there has or has not been spread to the axilla and the number of involved axillary nodes [1]. Several theories exist concerning the mechanism of breast cancer cell invasion and metastasis. Initially it was suggested that breast cancer first spreads locoregionally via lymphatics to the axillary lymph nodes and then metastasises more distantly. In accordance with this concept, Halsted developed radical mastectomy as the gold standard for breast cancer surgery [2].

Subsequently Fisher postulated that the extent of micrometastases at diagnosis of breast cancer is an indicator of outcome, with biological behaviour of cancer predetermining the likelihood of progression of the disease [3]. Nowadays gene expression profiling arrays can delineate tumour types with different prognoses [4]. The surgical approach for breast cancer treatment evolved from the extensive radical mastectomy and the Patey modified radical mastectomy [5] to breast conserving and minimally invasive techniques [6].

Traditionally the surgical management of breast cancer comprised wide local resection of the primary tumour and axillary lymph node dissection (ALND). Axillary status is the most important prognostic factor in breast cancer providing staging information and therefore largely defining treatment strategy [7]. Diagnostic imaging modalities such as ultrasound, magnetic resonance mammography, positron emission tomography, and $99 \mathrm{~m}$ Technetium (Tc) sestamibi scintimammography are not reliable for staging the axilla, particularly with lymph node metastases $<0.5 \mathrm{~cm}[8,9]$.

Clinical, pathological, and molecular features are inadequate for assessing ALN metastases. Clinically palpable lymph nodes prove to be false positive in $25-30 \%$ of patients [10] and about $40 \%$ have positive results after ultrasound with or without fine-needle aspiration node negativity [11]. Tumour size cannot serve as an accurate prognostic indicator for lymph node involvement. Studying 24,740 women with invasive breast cancer, Carter et al. showed that approximately $80 \%$ with tumour size $<1 \mathrm{~cm}, 50 \%$ with up to $5 \mathrm{~cm}$, and $30 \%$ with $>5 \%$ had negative axilla, a fact suggesting that metastases do not occur exclusively via the axillary lymph nodes, but rather lymph node status serves as an indicator of the tumour's ability to spread [12]. Additionally, it has been recently shown that the molecular profile of the primary tumour is a more significant prognostic indicator in terms 
of disease-free survival (DFS) and overall survival (OS) than lymph node metastases [4].

The combination of the introduction of populationbased mammographic screening for breast cancer, modern imaging methods, and increased public awareness resulted in patients being diagnosed more often with smaller-size tumours and less likelihood of axillary lymph node metastases [13]. It is evident now that $60-70 \%$ of patients with early breast cancer are node negative at the time of diagnosis [14] and ALND puts them at significant risk of short- and long-term morbidities without benefit [15]. ALND is associated with acute complication rates of 20 $30 \%$ including seroma formation, local swelling, numbness, impaired shoulder movement, neuropathy, infection, and chronic lymphoedema rates of $7-37 \%$ [16]. In a prospective study by Petrek et al. evaluating a cohort of 923 women with 20 years follow up, it was shown that breast-cancer-related lymphoedema following ALND occurred maximally in the first 3 years following surgery; however, up to $23 \%$ of patients may still develop arm swelling during the rest of their lives [17].

Several randomised studies have established that sentinel node biopsy ( $\mathrm{SNB}$ ) is a safe and accurate procedure for detecting tumour cells in SLN and predicting the status of the other axillary nodes (non-SLN). Although accuracy and appropriateness of SNB were disputed by the finding of 5$10 \%$ false-negative cases when SNB was followed by axillary dissection at high-risk patients for axillary nodal disease $[13,18]$, false-negative SNB results seem to have decreased with the increasing experience of surgeons, and it is expected that the utilisation of SNB in the future will be increased [19].

A meta-analysis of seven prospective randomised controlled trials by Kell et al. demonstrated that SNB is equivalent to ALND for the detection of lymph node metastasis with the additional advantage of reduction of up to $75 \%$ in morbidity in patients with early stage breast cancer. Furthermore, a trend towards an improved detection of LN metastases was shown when SNB is used [20]. Patients undergoing SNB have a $22 \%$ higher odds ratio of having a positive SLN, due to the more intensive pathological examination which utilises multiple sections and immunohistochemistry (IHC) [21]. In contrast, the false-negative cases seen after axillary dissection are probably due to the inability of the pathologist to perform serial sections and IHC on the 20-30 lymph nodes found in a complete axillary clearance specimen.

Studies have shown that SLN is the only positive lymph node in $38-67 \%$ of patients when ALNC followed [22]. Interestingly, it has been reported that only $4-8 \%$ of patients with negative ALNs have internal mammary lymph node involvement (IMN) whereas $25-50 \%$ of patients with affected ALNs have also IMN metastases [23, 24]. Dissection of IMN is not recommended because of the high morbidity and the uncertain benefit on survival [24].

The recently published outcomes of NSABP B-32 trial established the efficacy of SLN biopsy alone with no further ALND in 5611 breast cancer patients with clinically negative lymph nodes [25]. Women with invasive breast cancer who were randomly assigned to either SLN resection plus
ALND (group 1) or to SLN resection alone with ALND only if the SLNs were positive (group 2), after 8 years of followup, showed statistically equivalent overall survival, disease-free survival, and regional control. Patient followup is still continuing for longer-term assessment of survival and regional control.

Moreover, a closer look into mature studies focused on axillary relapses and overall survival is in agreement with current findings favouring SLB. The National Surgical Adjuvant Breast and Bowel Project (NSABP) B04 randomised study compared breast cancer patients with clinically negative ALNs managed either by radical mastectomy, total mastectomy with axillary radiation, or total mastectomy alone. The results clearly defined that ALND decreases the risk of locoregional relaps; however, no significant differences in survival were found among the treatment groups [26]. This study has been criticised because of the variability of numbers of lymph nodes resected in the total mastectomy alone arm and the lack of statistical power to detect a small difference in outcome [27]. Indeed, a meta-analysis has suggested that inadequate axillary treatment may lead to not only an increased risk of local relapse but also a $5 \%$ reduction in survival [28].

As a result of increasing detection of early breast cancer and the high rate of micrometastases and ITCs (ITCs) found in the detailed pathological examination of SLN, a new debate has opened about the consequent necessity of ALND in these patients. This has arisen because of better understanding of breast cancer behaviour and improved efficacy of combined therapeutic modalities. In this paper we report the current guidelines concerning the management of the axilla after SLNB and review the different aspects arising from recent studies on the role of micrometastases and ITC clusters in SLN on decision making.

\section{Current Guidelines}

The American Society of Clinical Oncology (ASCO) Expert Panel conducted a systematic review of the literature available through February 2004 on the use of SNB in earlystage breast cancer in order to develop guidelines for the management of the axilla (http://jop.ascopubs.org/content/ 1/4/134) and these are similar to those of the National Institute of Health and Clinical Excellence (NICE) recommendations in UK (http://www.nice.org.uk/nicemedia/ live/12132/43413/43413.pdf) [29].

SLNB is recommended for staging patients with clinically negative lymph nodes. ALND is the standard of care in those with a macrometastatic or micrometastatic positive SLN to maximise local control [30]. If the SLN is negative, a cALND (cALND) is not necessary. ITCs detected by IHC are of unknown clinical significance, and when identified, the SLN is regarded as negative and no further ALND is required. Although IHC is often used, it is not included in routine SLN evaluation for breast cancer at this time. ASCO and NICE recommendations for SLNB, ALND alone and managing of the axilla after SLNB are summarised in Table 1. In contrast the German guidelines do not recommend axillary clearance 
for 1-2 SLN positive in patients with T1 and T2 tumours (http://www.ago-online.de).

It has been suggested that SLNB should be carried out by an experienced team in order to minimise false negativity and improve the predictive value of the procedure [30]. All suspicious palpable nodes should also be considered as SLNs.

\section{Micrometastases and Isolated Tumour Cells (ITCs)}

The American Joint Committee on Cancer (AJCC) in the sixth edition of the Cancer Staging Manual defined a lymph node metastatic tumour with maximum diameter $>2 \mathrm{~mm}$ as macrometastasis ( $\mathrm{pN} 1)$, when the diameter of deposit is $0.2-2 \mathrm{~mm}$ as micrometastasis ( $\mathrm{pNmi}$ ), and a lesion of single tumour cells or small cell clusters with diameter $<0.2 \mathrm{~mm}$ as ITCs $[\mathrm{pN} 0(\mathrm{i}+)]$ [31]. ITCs are not distinguishable by $\mathrm{H} \& \mathrm{E}$ staining but detected only with immunohistochemistry (IHC) or molecular methods. Moore et al. suggested that the presence of ITCs was unrelated to known prognostic variables and partly the result of instrumentation and manipulation of the tumour [32].

The management of patients with minimal SLN involvement is problematic [33]. In a meta-analysis of 25 studies of patients with SLN micrometastases, in approximately $20 \%$ there was nonsentinel node disease falling to $9 \%$ when the SLN involvement was detected by IHC [34]. Furthermore, the consequent effect on DFS and OS remains controversial, so the biological relevance and clinical significance is a matter of debate [35].

AMAROS investigates the benefit of a cALND in comparison to treatment with axillary radiotherapy (ART) in patients with SLN-positive breast cancer [36]. A recently published substudy evaluated the identification rate and the nodal involvement of the first 2,000 patients between 2001 and 2005 who entered from 26 European institutions [36]. The sentinel node identification rate was $97 \%$ which is high considering the relatively early days of this procedure. $34 \%$ were SLN positive of whom $63 \%$ had macrometastases, $25 \%$ had micrometastases, and $12 \%$ had ITCs. In the cALND arm non-SLN involvement was identified in $41 \%$ of patients with macrometastases and in $18 \%$ of patients with either micrometastases or ITCs.

Several studies have investigated the significance of occult metastases, such as micrometastases or small clusters of tumour cells in association with non-SLN involvement and the impact of CALND on disease-free survival and overall survival, and the larger ones are summarised in Table 2 [37-43]. Although the majority show no prognostic impact of ITCs in the sentinel node, a large Dutch investigation with 5-year followup indicated that women with ITCs who received adjuvant chemotherapy had a significantly better event-free survival compared with untreated cases with ITCs [39]. Furthermore, a Finnish study showed a worse 5-year breast-cancer-specific survival for those with ITC compared with node negative cases [42].
In the largest published multicenter retrospective study of 187 SLN-ITCs patients undergoing cALND, Houvenaeghel et al. reported an incidence of $16 \%$ non-SLN involvement [44]. The difference in the risk of non-SLN involvement between sentinel nodes with ITCs (16\%) and those with micrometastases (14\%) was not statistically significant. However it was not apparent whether the presence of nonSLN metastases should affect the therapeutic decision in these patients. The authors proposed that cALND could be avoided in patients with tubular, colloid, or medullary small primary tumours ( $\mathrm{pT} 1)$ with a risk of non-SLN involvement approximately $\leq 5 \%$.

In contrast to the conclusions of the aforementioned studies come the MIRROR trial results [45]. MIRROR is a large Dutch cohort retrospective study which assesses the impact of SLN-ITCs and micrometastases on 5-year diseasefree survival in patients with favourable primary tumour characteristics. According to recent published data, both patients with SNB micrometastases and those with ITCs who did not undergo cALND experienced a far higher 5-year axillary recurrence rate, $6 \%$ in comparison to $1 \%$ of SNBnegative patients who did not undergo cALND. Additionally, both patients with SLN micrometastases and ITCs had approximately 5 -year disease-free survival improved by $10 \%$ with adjuvant systemic therapy. It is important to mention that micrometastases and ITCs had comparable prognostic impact [46]. MIRROR findings support an aggressive treatment approach in patients with either SLN micrometastases or ITCs.

\section{Completion ALND and Micrometastases}

Many investigators have studied the incidence of nonSLN involvement in patients with SLN micrometastases to define which patients may need further axillary treatment. Wada and Imoto. collected 22 studies from 1999 until 2006 referring to the frequency of SLN micrometastases in patients with breast cancer and the prevalence of nonSLN involvement in those patients after ALND [47]. The frequency of SLN micrometastases was 38\% with non-SLN micrometastases ranging from 0 to $57 \%$. Additionally, a wide range of non-SLN macrometastases was found (0-18\%). Because the prevalence of non-SLN micrometastases was low, the prognostic impact was unclear. The wide range of results arose from the different numbers of patients involved, variations in number of pathological sections examined, and differences in tumour stage and grade.

Results of studies in which patients with micrometastases in SNB and who were not treated by completion axillary node clearance are summarised in Table 3 [38, 48-54]. Most of the studies had small numbers and relatively short followup and tended to conclude that there was no benefit from completion axillary node clearance. The largest study; however, found a significantly worse disease-free survival for women with micrometastases who did not undergo cALND. [38].

De Boer et al. conducted a systematic review of 58 studies conducted from 1977 to 2008 included 297,533 patients, 
TABLE 1: Recommendations of SLNB, ALND, and treatment after SLNB.

\begin{tabular}{lccc}
\hline SLNB & ALND & \multicolumn{1}{c}{ Post-SLNB } \\
\hline T1, T2 tumour & T3, T4 tumours & SLNB +ve & ALND \\
Multicentric tumour & Inflammatory carcinoma & SLNB - ve & Observe \\
DCIS for mastectomy & Suspicious axillary node & Micromets & ALND \\
DCIS $>5 \mathrm{~cm}$ & Pregnancy & ITC & Observe \\
Older patient & Prior axillary surgery & SLNB +ve, 1-2 nodes, T1, T2 & Observe* \\
Preneoadjuvant & & & \\
\hline
\end{tabular}

DCIS: ductal carcinoma-in-situ; SNB: sentinel lymph node biopsy; ALND: axillary lymph node dissection.

* Recommendation of the German guidelines.

TABLE 2: Incidence and prognostic impact of ITCs in sentinel node biopsies.

\begin{tabular}{lccc}
\hline Author & Total & ITC (\%) & Outcome \\
\hline Herbert et al. [37] & 514 & $16(3 \%)$ & No effect \\
Reed et al. [38] & 1255 & $25(2 \%)$ & No effect \\
De Boer et al. [39] & 2707 & 819 & HR 1.5 (No adjuvant versus adjuvant) \\
Barbosa et al. [40] & 1000 & $43(4 \%)$ & No effect \\
Andersson et al. [41] & 3369 & $107(3 \%)$ & No effect \\
Leidenius et al. [42] & 1390 & $63(5 \%)$ & Reduced 5-year survival \\
Maaskant-Braat et al. [43] & 6803 & $126(2 \%)$ & No effect \\
\hline
\end{tabular}

aiming to define the prognostic relevance of micrometastases and ITCs in patients with breast cancer [55]. Using randomeffect meta-analysis they showed that the presence of ALN metastases $<2 \mathrm{~mm}$ in diameter detected on single-section examination was associated with poorer overall survival. Moreover the presence of occult metastases on retrospective examination of ALN-negative patients by step sectioning and/or immunohistochemistry ( $n=7740$ patients) was associated with poorer 5-year disease-free and overall survival. Outcomes from sentinel lymph node biopsy studies were not assessable due to small patient groups and short followup.

The International Breast Cancer Study Group trial IBCSG-23-01 is a randomised multicentre study designed to determine the significance of minimal LN metastasis in patients with breast cancer [56]. The trial was initiated in April 2001, and it compares survival between patients with SLN micrometastases who undergo SLNB alone with those who receive $\mathrm{CALND}$.

\section{Completion Axillary Lymph Node Dissection in SLN Macrometastases}

It is known that the extent of macrometastases in SLN is strongly correlated with non-SLN involvement. The longterm effect of the residual axillary disease in the sentinellymph-node-positive patient on local and systemic recurrence has not been clearly defined for patients receiving modern radiotherapy and chemotherapy. Older studies of patients with symptomatic breast cancers have shown that inadequate axillary surgery does lead to reduced overall survival [57-60].

Recently, the first results of the multicentre Z0011 trial were published [61]. The study set out to randomise
1900 women with breast cancer and 1-5 involved SNLN to either cALND or observation. All had a lumpectomy and tangential breast irradiation, but systemic therapy was at the discretion of the treating centre. After a median followup of 6.3 years, the relapse-free survival for the ALND group was $82 \%$ compared with $84 \%$ for the observation group, and the overall survival was $92 \%$ in both groups. Unfortunately the trial stopped accrual after 891 cases had been entered which makes it underpowered to detect a 5\% difference in outcome. An additional aspect to be considered is that the Guy's wide excision studies showed no difference between the wide excision group and the radical mastectomy group at 10 years whereas after 25 years there was a significantly worse relapse-free and overall survival in the wide excision group with inadequately treated axillae [58].

In a retrospective study, Takei et al. confirmed the importance of cALND in SLN-positive patients with high nuclear grade and hormone-negative breast cancer. It was noticed that of 459 patients with macrometastatic disease treated with CALND, after a median follow-up period of 34 months, the axillary recurrence rate was only $0.6 \%$ [62]. Bilimoria et al. studied a cohort of 403,167 patients with clinically node-negative breast cancer that underwent SLNB from the US National Cancer Data Base (1998-2005) [63]. Of the $97,314(24 \%)$ patients identified with nodal metastases, $28 \%$ had no further surgical intervention in the axilla and $72 \%$ underwent cALND. After a median followup of 63 months, it was found that in all patients and separately in those with macroscopic and microscopic nodal disease, the unadjusted axillary recurrence rate and overall survival were comparable. After adjustment for clinicopathological differences, there was a trend towards a lower risk of axillary recurrence and death in patients with macroscopic nodal 
TABLE 3: Studies of patients with micrometastases not treated by completion mastectomy.

\begin{tabular}{lccc}
\hline Author & Total & Follow-up (months) & Outcome \\
\hline Fan et al. [48] & 27 & 17 & 1 recurrence \\
Nagashima et al. [49] & 19 & 24 & 1 recurrence \\
Yegiyants et al. [50] & 33 & 84 & 1 recurrence \\
Fournier et al. [51] & 16 & 30 & No recurrence \\
Langer et al. [52] & 27 & 77 & No recurrence \\
Meretoja et al. [53] & 48 & 37 & 3 recurrences, 1 death \\
Pernas et al, [54] & 45 & 60 & 1 recurrence \\
Reed et al. [38] & 57 & 59 & Significantly reduced disease-free interval \\
\hline
\end{tabular}

involvement undergoing cALND. For those with micrometastases, recurrence rates were similar in those undergoing either SLNB alone or CALND.

Of 26,986 patients with SLNB-positive breast cancer from the SEER database (surveillance, epidemiology, and end results), $16 \%$ had no further axillary treatment and $84 \%$ had cALND [64]. After a median followup of 50 months, although a higher rate of ipsilateral regional recurrence was noticed in patients who underwent SLNB alone, no statistically significant differences in overall survival (OS) between patients who underwent SLNB alone versus complete ALND were found. The investigators suggested that in patients with small, low-grade primary tumours, positive ER status, older age and who have received segmental mastectomy, cALND may be omitted [64].

Hwang et al. reviewed the outcome of 3,366 patients with invasive breast cancer who underwent SLNB from 1993 to 2005 [65]. Of 750 SLN-positive patients, 65\%, 45.9\%, and $34.2 \%$ were $\mathrm{pN} 1, \mathrm{pN} 1 \mathrm{mi}$, and $\mathrm{pN} 0$ (i+), respectively. Of these patients, 196 had no further axillary surgery due to clinician and patient preference. According to clinicopathological variables, adjuvant treatment was applied and locoregional and distant recurrence and survival were studied. After a median followup of 29.5 months, no patient had an axillary recurrence, one had supraclavicular lymph node recurrence, and three patients developed metastatic disease to the lung or bone. The median time to recurrence was 32 months. Notably the patients with distant metastases had T3 grade III invasive carcinoma. Despite the low axillary recurrence rate, authors suggested that it is not possible from these results to conclude definitively that cALND should be abandoned for these patients [65].

\section{Predictive Models}

Several factors correlated with the likelihood of additional non-SLN metastasis have been investigated in an effort to distinguish which patients could avoid extensive axillary surgery. Characteristics of the primary tumour, such as size $[40,66]$, grade [67], hormone receptor and HER2 profile [67], tumour type [67], multifocality, mean proliferative fraction, and lymphovascular invasion $[68,69]$, have all been studied. Additional features of the involved SLNs, such as size of metastases [40], number of positive SLNs [40], ratio of positive to resected SLNs, and the extracapsular spread have
TABLE 4: Major prognostic factors for non-SLN metastases in patients with minimal SLN metastases.

\begin{tabular}{ll}
\hline Feature & Author \\
\hline $\begin{array}{l}\text { Lymphovascular } \\
\text { invasion }\end{array}$ & $\begin{array}{l}\text { Mittendorf et al. [67], } \\
\text { Van Deurzen et al. [66], } \\
\text { Viale et al. [69], } \\
\text { Jinno et al. [68] }\end{array}$ \\
\hline $\begin{array}{l}\text { Size of SLN } \\
\text { metastases }\end{array}$ & $\begin{array}{l}\text { Barbosa et al. [40], } \\
\text { Van Deurzen et al. [66], } \\
\text { Primary tumour } \\
\text { size }\end{array}$ \\
\hline $\begin{array}{l}\text { Lobular } \\
\text { histology }\end{array}$ & Barbosa et al. [69] [40], \\
\hline $\begin{array}{l}\text { Number of } \\
\text { positive SLN }\end{array}$ & Van Deurzen et al. [66] \\
\hline
\end{tabular}

been also examined [21, 61, 70-72]. Particularly patients with minimal SLN metastases are at a significantly lower risk to have further non-SLN invasion than those with SLN macrometastases (13-24\% versus 45-79\%) [61]. However, none of these characteristics individually can determine a subset of patients for whom ALND is unnecessary. Molecular profiling of metastatic foci different from the primary tumour could be used as indicator for the selection of patients who might benefit of completion axillary dissection [73]. The most important prognostic factors for the presence of non-SLN metastases in patients with minimal SLN involvement are presented in Table 4.

Several mathematical models have been developed to predict the risk of non-SLN involvement in patients with SLN-positive breast cancer [74]. These include four nomograms: the Memorial Sloan-Kettering Cancer Center (MSKCC) [75], (https://www.mskcc.org/mskcc/html/15938 .cfm), Mayo [76] (http://www.mayoclinic.org/breast-cancer/ sentinelbiopsy.html), Cambridge [77], and Stanford [70] (https://www3-hrpdcc.stanford.edu/nsln-calculator/). There are three scoring systems, the Tenon, MDA, and Saidi, and two recursive partitioning (RP) tools developed by Kohrt et al. [70].

The Institut Curie studied 588 consecutive patients with positive SLNs who underwent ALND to compare the actual 
TABLE 5: Validation studies comparing OSNA with histopathology.

\begin{tabular}{lcccc}
\hline Author & Total & Concordance & Sensitivity & Specificity \\
\hline Tsujimoto et al. [71] & 101 & $98 \%$ & $91 \%$ & $100 \%$ \\
Schem et al. [72] & 93 & $92 \%$ & $98 \%$ & $91 \%$ \\
Tamaki et al. [79] & 185 & $93 \%$ & $88 \%$ & $94 \%$ \\
Snook et al. [80] & 204 & $96 \%$ & $92 \%$ & $97 \%$ \\
Feldman et al. [81] & 498 & $96 \%$ & $78 \%$ & $96 \%$ \\
\hline
\end{tabular}

rate of non-SLN metastases with those predicted by Breast Cancer Nomogram of Memorial Sloan-Kettering Cancer Center (MSKCC). While the predicted rate in non-SLN macrometastases was relatively accurate, when the nomogram was applied to the 213 SLNs that contained only micrometastases, the predicted rate $5-9 \%$ was far away from the actual rate $44 \%$ of non-SLN micrometastases detect-ed by IHC. Consequently, the authors concluded that a different predictive model should be created for patients with micrometastases.

Molecular tests based on technology such as Oncotype Dx (Genomic Health, Redwood City, Calif, USA) or other multigene arrays developed prognostic and predictive markers aiming to personalize surgical and adjuvant treatment of early breast cancer [4]. An accurate, intraoperative sentinel lymph node test probably could help in avoidance of delayed axillary dissections. Molecular tests may be proved more sensitive than current intraoperative tests but have not yet been validated.

\section{One-Step Nucleic Acid Amplification (OSNA)}

OSNA is an automated assay for the detection of cytokeratin message, CK19 mRNA, present in approximately $98 \%$ of breast cancers [78]. It provides an opportunity to make an intraoperative diagnosis of sentinel node involvement within 30 minutes, avoiding frozen section and allowing a onestage procedure. Larger verification studies in which half of the bisected sentinel node was sent for histology and the other half homogenised for OSNA are shown in Table 5 [71, 72, 79-81]. The usual reason for discordance between histopathology and OSNA was an uneven distribution of nodal metastases (tissue allocation bias). This problem is abolished when the entire node is subjected to OSNA.

The studies indicate a good concordance between results of histopathology and OSNA. Indeed in a study conducted by Osaka, comparing OSNA with frozen section, the former was more sensitive, increasing the positive sentinel node rate by $30 \%$ [82]. It is likely that in time OSNA will replace histopathological examination of sentinel lymph nodes because of its ease, accuracy, and potential for enabling almost all patients to have a one-stage operation for early breast cancer.

\section{Conclusions}

Recent studies aiming to determine if cALND is beneficial in patients with SLN-positive breast cancer and even more in patients with minimal SLN involvement have reached contrary conclusions. Limitations in the published studies include the methods of pathological evaluation of lymph nodes and that the number of additional non-SLN-positive nodes is usually not known. Detection rate of micrometastases and ITCs depends on histopathological technique and protocol. Thus lymph node step sectioning and IHC lead to increased identification of minimal metastases upstaging $9 \%$ to $25 \%$ of patients who initially were considered node negative $[83,84]$ and did not have further axillary surgery.

The different rates of recurrence may be due to the molecular type of cancers and so that patients with SLN metastases may also have different risks of metastatic involvement [40]. Finally it is possible that not all minor tumour foci in axillary lymph nodes progress to local recurrences. According to Al-Hajj et al., only a minority of cancer cells potentially give metastases and most ITCs are not viable and do not have the ability to form new tumours [85]. There may be two different breast cancer cell populations, true stem cells that have the capacity to develop metastases and the nonstem cells that never grow and are finally destroyed [86].

At the level of everyday clinical practice, with both promising and disappointing results of the published studies, most breast surgeons will hardly ever take the risk of avoiding completion axillary dissection in breast cancer patients even with minimal sentinel lymph node metastases. Many are seeking to find a balance between the needs of the majority to have minimal axillary surgery with minimal postoperative morbidity against the possibility that a minority will suffer relapse, morbidity, and possible increased mortality from undertreatment. Results from ongoing phase III trials will perhaps provide new guidelines for the treatment of patients with micrometastases or ITCs. A predictive model which estimates accurately the likelihood of additional disease in the axilla might help tailor surgical therapy to the needs of the individual patient and identify those most likely to benefit from completion or ALND. Genetic assays defining prognostic markers and new intraoperative tests detecting accurately SLN involvement will help in early therapeutic decision making in the future. It is important that premature decisions to restrict axillary surgery are not made on a basis of early results from underpowered clinical trials.

\section{References}

[1] T. Nemoto, J. Vana, and R. N. Bedwani, "Management and survival of female breast cancer: results of a national survey by the American college of surgeons," Cancer, vol. 45, no. 12, pp. 2917-2924, 1980.

[2] W. Halsted, "The results of operations for the cure of cancer of the breast performed at the Johns Hopkins Hospitals from June 1889 to January 1894," Bulletin of the Johns Hopkins Hospital, vol. 4, pp. 297-302, 1895.

[3] B. Fisher, "A biological basis for the surgical treatment of clinically curable breast cancer," Bulletin de la Societe internationale de chirurgie, vol. 31, no. 6, pp. 604-609, 1972.

[4] L. J. Van't Veer, H. Dai, M. J. Van de Vijver et al., "Gene expression profiling predicts clinical outcome of breast cancer," Nature, vol. 415, no. 6871, pp. 530-536, 2002. 
[5] D. H. Patey and W. H. Dyson, "The prognosis of carcinoma of the breast in relation to the type of operation performed," British Journal of Cancer, vol. 2, no. 1, pp. 7-13, 1948.

[6] J. A. Van Dongen, H. Bartelink, I. S. Fentiman et al., "Factors influencing local relapse and survival and results of salvage treatment after breast-conserving therapy in operable breast cancer: EORTC trial 10801, breast conservation compared with mastectomy in TNM Stage I and II breast cancer," European Journal of Cancer, vol. 28, no. 4-5, pp. 801-805, 1992.

[7] S. Samphao, J. M. Eremin, M. El-Sheemy, and O. Eremin, "Management of the axilla in women with breast cancer: current clinical practice and a new selective targeted approach," Annals of Surgical Oncology, vol. 15, no. 5, pp. 1282-1296, 2008.

[8] N. R. Patani, M. V. Dwek, and M. Douek, "Predictors of axillary lymph node metastasis in breast cancer: a systematic review," European Journal of Surgical Oncology, vol. 33, no. 4, pp. 409-419, 2007.

[9] R. L. Wahl, B. A. Siegel, R. E. Coleman, and C. G. Gatsonis, "Prospective multicenter study of axillary nodal staging by positron emission tomography in breast cancer: a report of the staging breast cancer with PET study group," Journal of Clinical Oncology, vol. 22, no. 2, pp. 277-285, 2004.

[10] M. C. Specht, J. V. Fey, P. I. Borgen, and H. S. Cody, "Is the clinically positive axilla in breast cancer really a contraindication to sentinel lymph node biopsy?" Journal of the American College of Surgeons, vol. 200, no. 1, pp. 10-14, 2005.

[11] E. E. Deurloo, P. J. Tanis, K. G. A. Gilhuijs et al., "Reduction in the number of sentinel lymph node procedures by preoperative ultrasonography of the axilla in breast cancer," European Journal of Cancer, vol. 39, no. 8, pp. 1068-1073, 2003.

[12] C. L. Carter, C. Allen, and D. E. Henson, "Relation of tumor size, lymph node status, and survival in 24,740 breast cancer cases," Cancer, vol. 63, no. 1, pp. 181-187, 1989.

[13] U. Veronesi, G. Paganelli, G. Viale et al., "A randomized comparison of sentinel-node biopsy with routine axillary dissection in breast cancer," The New England Journal of Medicine, vol. 349, no. 6, pp. 546-553, 2003.

[14] M. R. Kell and M. J. Kerin, "Sentinel lymph node biopsy," British Medical Journal, vol. 328, no. 7452, pp. 1330-1331, 2004.

[15] B. Cady, M. D. Stone, J. G. Schuler, R. Thakur, M. A. Wanner, and P. T. Lavin, "The new era in breast cancer: invasion, size, and nodal involvement dramatically decreasing as a result of mammographic screening," Archives of Surgery, vol. 131, no. 3, pp. 301-308, 1996.

[16] A. E. Giuliano, P. I. Haigh, M. B. Brennan et al., "Prospective observational study of sentinel lymphadenectomy without further axillary dissection in patients with sentinel node-negative breast cancer," Journal of Clinical Oncology, vol. 18, no. 13, pp. 2553-2559, 2000.

[17] J. A. Petrek, R. T. Senie, M. Peters, and P. Peterrosen, "Lymphedema in a cohort of breast carcinoma survivors 20 years after diagnosis," Cancer, vol. 92, no. 6, pp. 1368-1377, 2001.

[18] R. E. Mansel, L. Fallowfield, M. Kissin et al., "Randomized multicenter trial of sentinel node biopsy versus standard axillary treatment in operable breast cancer: the ALMANAC trial," Journal of the National Cancer Institute, vol. 98, no. 9, pp. 599609, 2006.

[19] J. M. Classe, C. Curtet, L. Campion et al., "Learning curve for the detection of axillary sentinel lymph node in breast cancer," European Journal of Surgical Oncology, vol. 29, no. 5, pp. 426433, 2003.

[20] M. R. Kell, J. P. Burke, M. Barry, and M. Morrow, "Outcome of axillary staging in early breast cancer: a meta-analysis," Breast
Cancer Research and Treatment, vol. 120, no. 2, pp. 441-447, 2010.

[21] A. J. G. Maaskant, L. V. Van De Poll-Franse, A. C. Voogd, J. W. W. Coebergh, M. C. B. J. E. Tutein Nolthenius-Puylaert, and G. A. P. Nieuwenhuijzen, "Stage migration due to introduction of the sentinel node procedure: a population-based study," Breast Cancer Research and Treatment, vol. 113, no. 1, pp. 173-179, 2009.

[22] C. Reynolds, R. Mick, J. H. Donohue et al., "Sentinel lymph node biopsy with metastasis: can axillary dissection be avoided in some patients with breast cancer?" Journal of Clinical Onco$\log y$, vol. 17, no. 6, pp. 1720-1726, 1999.

[23] N. Klauber-DeMore, J. L. B. Bevilacqua, K. J. Van Zee, P. Borgen, and H. S. Cody, "Comprehensive review of the management of internal mammary lymph node metastases in breast cancer," Journal of the American College of Surgeons, vol. 193, no. 5, pp. 547-555, 2001.

[24] U. Veronesi, E. Marubini, L. Mariani, P. Valagussa, and R. Zucali, "The dissection of internal mammary nodes does not improve the survival of breast cancer patients. 30-Year results of a randomised trial," European Journal of Cancer, vol. 35, no. 9, pp. 1320-1325, 1999.

[25] D. N. Krag, S. J. Anderson, T. B. Julian et al., "Sentinel-lymphnode resection compared with conventional axillary-lymphnode dissection in clinically node-negative patients with breast cancer: overall survival findings from the NSABP B-32 randomised phase 3 trial," The Lancet Oncology, vol. 11, no. 10, pp. 927-933, 2010.

[26] B. Fisher, J. H. Jeong, S. Anderson, J. Bryant, E. R. Fisher, and N. Wolmark, "Twenty-five-year follow-up of a randomized trial comparing radical mastectomy, total mastectomy, and total mastectomy followed by irradiation," The New England Journal of Medicine, vol. 347, no. 8, pp. 567-575, 2002.

[27] J. R. Harris and R. T. Osteen, "Patients with early breast cancer benefit from effective axillary treatment," Breast Cancer Research and Treatment, vol. 5, no. 1, pp. 17-21, 1985.

[28] R. K. Orr, "The impact of prophylactic axillary node dissection on breast cancer survival - A Bayesian meta-analysis," Annals of Surgical Oncology, vol. 6, no. 1, pp. 109-116, 1999.

[29] J. Yarnold, "Early and locally advanced breast cancer: diagnosis and treatment National Institute for Health and Clinical Excellence Guideline 2009," Clinical Oncology, vol. 21, no. 3, pp. 159-160, 2009.

[30] G. H. Lyman, A. E. Giuliano, M. R. Somerfield et al., "American Society of Clinical Oncology guideline recommendations for sentinel lymph node biopsy in early-stage breast cancer," Journal of Clinical Oncology, vol. 23, no. 30, pp. 7703-7720, 2005.

[31] S. E. Singletary and F. L. Greene, "Revision of breast cancer staging: the 6th edition of the TNM Classification," Seminars in Surgical Oncology, vol. 21, no. 1, pp. 53-59, 2003.

[32] K. H. Moore, H. T. Thaler, L. K. Tan, P. I. Borgen, and H. S. Cody, "Immunohistochemically detected tumor cells in the sentinel lymph nodes of patients with breast carcinoma: biologic metastasis or procedural artifact?" Cancer, vol. 100, no. 5, pp. 929-934, 2004.

[33] I. De Mascarel, F. Bonichon, J. M. Coindre, and M. Trojani, "Prognostic significance of breast cancer axillary lymph node micrometastases assessed by two special techniques: reevaluation with longer follow-up," British Journal of Cancer, vol. 66, no. 3, pp. 523-527, 1992.

[34] G. Cserni, D. Gregori, F. Merletti et al., "Meta-analysis of nonsentinel node metastases associated with micrometastatic sen- 
tinel nodes in breast cancer," British Journal of Surgery, vol. 91, no. 10, pp. 1245-1252, 2004.

[35] G. Cserni, I. Amendoeira, N. Apostolikas et al., "Pathological work-up of sentinel lymph nodes in breast cancer. Review of cur-rent data to be considered for the formulation of guidelines," European Journal of Cancer, vol. 39, no. 12, pp. 16541667, 2003.

[36] M. E. Straver, P. Meijnen, G. Van Tienhoven et al., "Sentinel node identification rate and nodal involvement in the EORTC 10981-22023 AMAROS trial," Annals of Surgical Oncology, vol. 17, no. 7, pp. 1854-1861, 2010.

[37] G. S. Herbert, V. Y. Sohn, and T. A. Brown, "The impact of nodal isolated tumor cells on survival of breast cancer pa-tients," American Journal of Surgery, vol. 193, no. 5, pp. 571-574, 2007.

[38] J. Reed, M. Rosman, K. M. Verbanac, A. Mannie, Z. Cheng, and L. Tafra, "Prognostic implications of isolated tumor cells and micrometastases in sentinel nodes of patients with invasive breast cancer: 10-Year analysis of patients enrolled in the prospective East Carolina University/Anne Arundel Medical Center Sentinel Node Multicenter Study," Journal of the American College of Surgeons, vol. 208, no. 3, pp. 333-340, 2009.

[39] M. De Boer, C. H. M. Van Deurzen, J. A. A. M. Van Dijck et al., "Micrometastases or isolated tumor cells and the outcome of breast cancer," The New England Journal of Medicine, vol. 361, no. 7, pp. 653-663, 2009.

[40] E. M. Barbosa, A. A. R. F. Francisco, J. T. Araujo Neto, E. M. F. Alves, M. G. M. Tavares, and J. C. S. Góes, "Clinicopathological predictor factors of axillary involvement in patients with metastatic breast cancer in the sentinel lymph node," Revista Brasileira de Ginecologia e Obstetricia, vol. 32, no. 3, pp. 144$149,2010$.

[41] Y. Andersson, J. Frisell, M. Sylvan, J. de Boniface, and L. Bergkvist, "Breast cancer survival in relation to the metastatic tumor burden in axillary lymph nodes," Journal of Clinical Oncology, vol. 28, no. 17, pp. 2868-2873, 2010.

[42] M. H. K. Leidenius, J. H. Vironen, P. S. Heikkilä, and H. Joensuu, "Influence of isolated tumor cells in sentinel nodes on outcome in small, node-negative (pT1N0M0) breast cancer," Annals of Surgical Oncology, vol. 17, no. 1, pp. 254-262, 2010.

[43] A. J. Maaskant-Braat et al., "Sentinel node micrometastases in breast cancer do not affect prognosis: a population-based study," Breast Cancer Research and Treatment, vol. 127, no. 1, pp. 195-203, 2011.

[44] G. Houvenaeghel, C. Nos, H. Mignotte et al., "Micrometastases in sentinel lymph node in a multicentric study: predictive factors of nonsentinel lymph node involvement - Groupe des Chirurgiens de la Federation des Centres de Lutte Contre Le Cancer," Journal of Clinical Oncology, vol. 24, no. 12, pp. 18141822, 2006.

[45] V. C. Tjan-Heijnen et al., "Impact of omission of completion axillary lymph node dissection (cALND) or axillary radiotherapy (ax RT) in breast cancer patients with micrometastases (pN1mi) or isolated tumor cells $(\mathrm{pNO}[\mathrm{i}+])$ in the sentinel lymph node (SN): results from the MIRROR study," Journal of Clinical Oncology, vol. 27, abstract CRA506, p. 18s, 2009.

[46] V. C. Tjan-Heijnen and M. de Boer, "Minimal lymph node involvement and outcome of breast cancer. The results of the Dutch MIRROR study," Discovery Medicine, vol. 8, no. 42, pp. 137-139, 2009.

[47] N. Wada and S. Imoto, "Clinical evidence of breast cancer micrometastasis in the era of sentinel node biopsy," International Journal of Clinical Oncology, vol. 13, no. 1, pp. 24-32, 2008.
[48] Y. G. Fan, Y. Y. Tan, C. T. Wu et al., "The effect of sentinel node tumor burden on non-sentinel node status and recurrence rates in breast cancer," Annals of Surgical Oncology, vol. 12, no. 9, pp. 705-711, 2005.

[49] T. Nagashima, M. Sakakibara, S. Nakano et al., "Sentinel node micrometastasis and distant failure in breast cancer patients," Breast Cancer, vol. 13, no. 2, pp. 186-191, 2006.

[50] S. Yegiyants, L. M. Romero, P. I. Haigh, and L. A. DiFronzo, "Completion axillary lymph node dissection not required for regional control in patients with breast cancer who have micrometastases in a sentinel node," Archives of Surgery, vol. 145, no. 6, pp. 564-569, 2010.

[51] K. Fournier, A. Schiller, R. R. Perry et al., "Micrometastasis in the sentinel lymph node of breast cancer does not mandate completion axillary dissection," Annals of Surgery, vol. 239, no. 6, pp. 859-865, 2004.

[52] I. Langer, U. Guller, C. T. Viehl et al., "Axillary lymph node dissection for sentinel lymph node micrometastases may be safely omitted in early-stage breast cancer patients: long-term outcomes of a prospective study," Annals of Surgical Oncology, vol. 16, no. 12, pp. 3366-3374, 2009.

[53] T. J. Meretoja, J. H. Vironen, P. S. Heikkilä, and M. H. Leidenius, "Outcome of selected breast cancer patients with micrometastasis or isolated tumor cells in sentinel node biopsy and no completion axillary lymph node dissection," Journal of Surgical Oncology, vol. 102, no. 3, pp. 215-219, 2010.

[54] S. Pernas, M. Gil, A. Benítez et al., "Avoiding axillary treatment in sentinel lymph node micrometastases of breast cancer: a prospective analysis of axillary or distant recurrence," Annals of Surgical Oncology, vol. 17, no. 3, pp. 772-777, 2010.

[55] M. De Boer, J. A. A. M. Van Dijck, P. Bult, G. F. Borm, and V. C. G. Tjan-Heijnen, "Breast cancer prognosis and occult lymph node metastases, isolated tumor cells, and micrometastases," Journal of the National Cancer Institute, vol. 102, no. 6, pp. 410-425, 2010.

[56] V. Galimberti, "International Breast Cancer Study Group trial of sentinel node biopsy," Journal of Clinical Oncology, vol. 24, no. 1, pp. 210-211, 2006.

[57] D. Ziogas and G. C. Zografos, "Micrometastatic sentinel lymph node disease: do patients benefit or suffer harm from axillary lymph node dissection?” Annals of Surgical Oncology, vol. 17, no. 3, pp. 936-937, 2010.

[58] I. S. Fentiman, "Long-term follow-up of the first breast conservation trial: guy's wide excision study," Breast, vol. 9, no. 1, pp. 5-8, 2000.

[59] C. K. Axelsson, H. T. Mouridsen, and K. Zedeler, "Axillary dissection of level I and II lymph nodes is important in breast cancer classification. The Danish Breast Cancer Cooperative Group (DBCG)," European Journal of Cancer, vol. 28, no. 8-9, pp. 1415-1418, 1992.

[60] H. S. Shukla, J. Melhuish, R. E. Mansel, and L. E. Hughes, "Does local therapy affect survival rates in breast cancer?" Annals of Surgical Oncology, vol. 6, no. 5, pp. 455-460, 1999.

[61] A. E. Giuliano et al., "Axillary dissection vs no axillary dissection in women with invasive breast cancer and sentinel node metastasis. A randomized clinical trial," Journal of the American Medical Association, vol. 305, no. 6, pp. 569-575, 2011.

[62] H. Takei, K. Suemasu, M. Kurosumi et al., "Recurrence after sentinel lymph node biopsy with or without axillary lymph node dissection in patients with breast cancer," Breast Cancer, vol. 14, no. 1, pp. 16-24, 2007. 
[63] K. Y. Bilimoria, D. J. Bentrem, N. M. Hansen et al., "Comparison of sentinel lymph node biopsy alone and completion axillary lymph node dissection for node-positive breast cancer," Journal of Clinical Oncology, vol. 27, no. 18, pp. 2946-2953, 2009.

[64] M. Yi, S. H. Giordano, F. Meric-Bernstam et al., "Trends in and outcomes from sentinel lymph node biopsy (SLNB) alone vs. SLNB with axillary lymph node dissection for node-positive breast cancer patients: experience from the SEER database," Annals of Surgical Oncology, vol. 17, no. 3, pp. S343-S351, 2010.

[65] R. F. Hwang, A. M. Gonzalez-Angulo, M. Yi et al., "Low locoregional failure rates in selected breast cancer patients with tumor-positive sentinel lymph nodes who do not under-go completion axillary dissection," Cancer, vol. 110, no. 4, pp. 723-730, 2007.

[66] C. H. M. Van Deurzen, R. Van Hillegersberg, M. G. G. Hobbelink, C. A. Seldenrijk, R. Koelemij, and P. J. Van Diest, "Predictive value of tumor load in breast cancer sentinel lymph nodes for second echelon lymph node metastases," Cellular Oncology, vol. 29, no. 6, pp. 497-505, 2007.

[67] E. A. Mittendorf, A. A. Sahin, S. L. Tucker et al., "Lymphovascular invasion and lobular histology are associated with increased incidence of isolated tumor cells in sentinel lymph nodes from early-stage breast cancer patients," Annals of Surgical Oncology, vol. 15, no. 12, pp. 3369-3377, 2008.

[68] H. Jinno, M. Sakata, S. Asaga et al., "Predictors to assess nonsentinel lymph node status in breast cancer patients with sentinel lymph node metastasis," Breast Journal, vol. 14, no. 6, pp. 551-555, 2008.

[69] G. Viale, E. Maiorano, G. Pruneri et al., "Predicting the risk for additional axillary metastases in patients with breast carcinoma and positive sentinel lymph node biopsy," Annals of Surgery, vol. 241, no. 2, pp. 319-325, 2005.

[70] H. E. Kohrt, R. A. Olshen, H. R. Bermas et al., "New models and online calculator for predicting non-sentinel lymph node status in sentinel lymph node positive breast cancer patients," BMC Cancer, vol. 8, article no. 66, 2008.

[71] M. Tsujimoto, K. Nakabayashi, K. Yoshidome et al., "Onestep nucleic acid amplification for intraoperative detection of lymph node metastasis in breast cancer patients," Clinical Cancer Research, vol. 13, no. 16, pp. 4807-4816, 2007.

[72] C. Schem, N. Maass, D. O. Bauerschlag et al., "One-step nucleic acid amplification-a molecular method for the detection of lymph node metastases in breast cancer patients; Results of the German study group," Virchows Archiv, vol. 454, no. 2, pp. 203-210, 2009.

[73] M. Vecchi, S. Confalonieri, P. Nuciforo et al., "Breast cancer metastases are molecularly distinct from their primary tumors," Oncogene, vol. 27, no. 15, pp. 2148-2158, 2008.

[74] C. Coutant, C. Olivier, E. Lambaudie et al., "Comparison of models to predict nonsentinel lymph node status in breast cancer patients with metastatic sentinel lymph nodes: a prospective multicenter study," Journal of Clinical Oncology, vol. 27, no. 17, pp. 2800-2808, 2009.

[75] K. J. Van Zee, D. M. E. Manasseh, J. L. B. Bevilacqua et al., “A nomogram for predicting the likelihood of additional nodal metastases in breast cancer patients with a positive sentinel node biopsy," Annals of Surgical Oncology, vol. 10, no. 10, pp. 1140-1151, 2003.

[76] A. C. Degnim, C. Reynolds, G. Pantvaidya et al., "Nonsentinel node metastasis in breast cancer patients: assessment of an existing and a new predictive nomogram," American Journal of Surgery, vol. 190, no. 4, pp. 543-550, 2005.

[77] A. Pal, E. Provenzano, S. W. Duffy, S. E. Pinder, and A. D. Pumshotham, "A model for predicting non-sentinel lymph node metastatic disease when the sentinel lymph node is positive," British Journal of Surgery, vol. 95, no. 3, pp. 302-309, 2008.

[78] P. G. Chu and L. M. Weiss, "Keratin expression in human tissues and neoplasms," Histopathology, vol. 40, no. 5, pp. 403439, 2002.

[79] YasuhiroTamaki, F. Akiyama, T. Iwase et al., "Molecular detection of lymph node metastases in breast cancer patients: results of a multicenter trial using the one-step nucleic acid amplification assay," Clinical Cancer Research, vol. 15, no. 8, pp. 2879-2884, 2009.

[80] K. L. Snook et al., "Multicentre evaluation of intraoperative molecular analysis of sentinel lymph nodes in breast carcinoma," British Journal of Surgery, vol. 98, pp. 527-535, 2011.

[81] S. Feldman et al., "A novel automated assay for the rapid identification of metastatic breast carcinoma in sentinel lymph nodes," Cancer, vol. 117, no. 112, pp. 2599-2607, 2011.

[82] T. Osaka et al., "Intraoperative molecular assay for sentinel lymph node metastases in early stage breast cancer: a comparative analysis between one-step nucleic acid amplification whole node assay and routine frozen section histology," Cancer. In press.

[83] A. D. K. Hill, K. N. Tran, T. Akhurst et al., "Lessons learned from 500 cases of lymphatic mapping for breast cancer," Annals of Surgery, vol. 229, no. 4, pp. 528-535, 1999.

[84] D. L. Weaver, D. N. Krag, T. Ashikaga, S. P. Harlow, and M. O'Connell, "Pathologic analysis of sentinel and nonsentinel lymph nodes in breast carcinoma: a multicenter study," Cancer, vol. 88, no. 5, pp. 1099-1107, 2000.

[85] M. Al-Hajj, M. S. Wicha, A. Benito-Hernandez, S. J. Morrison, and M. F. Clarke, "Prospective identification of tumorigenic breast cancer cells," Proceedings of the National Academy of Sciences of the United States of America, vol. 100, no. 7, pp. 3983-3988, 2003.

[86] C. Colpaert, P. Vermeulen, W. Jeuris et al., "Early distant relapse in 'node-negative' breast cancer patients is not predicted by occult axillary lymph node metastases, but by the features of the primary tumour," Journal of Pathology, vol. 193, no. 4, pp. 442-449, 2001. 


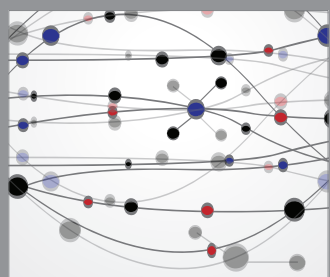

The Scientific World Journal
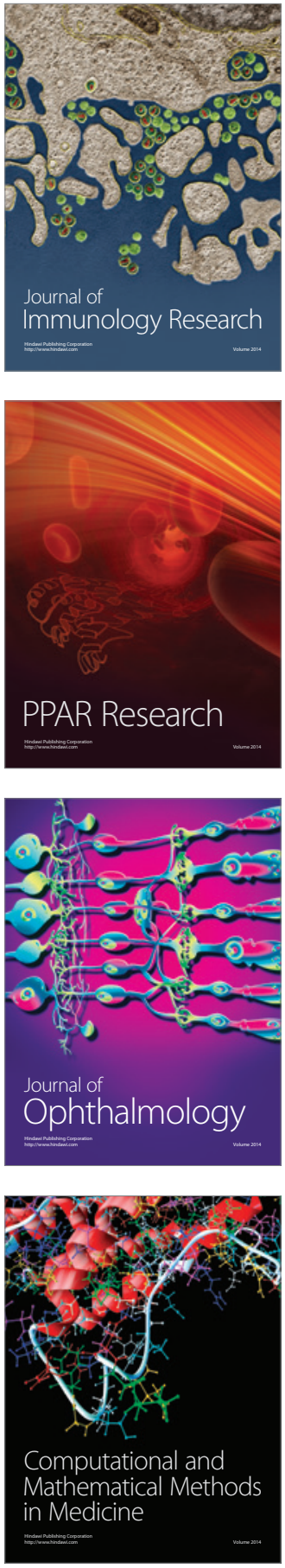

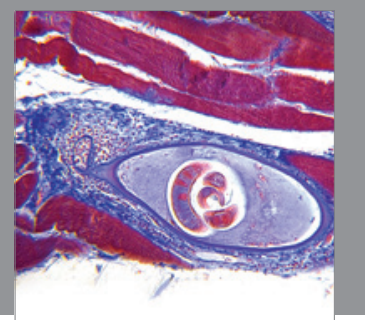

Gastroenterology

Research and Practice
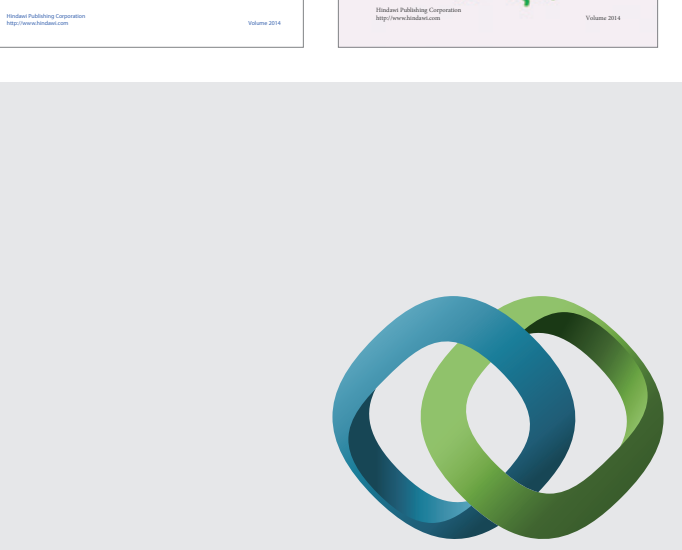

\section{Hindawi}

Submit your manuscripts at

http://www.hindawi.com
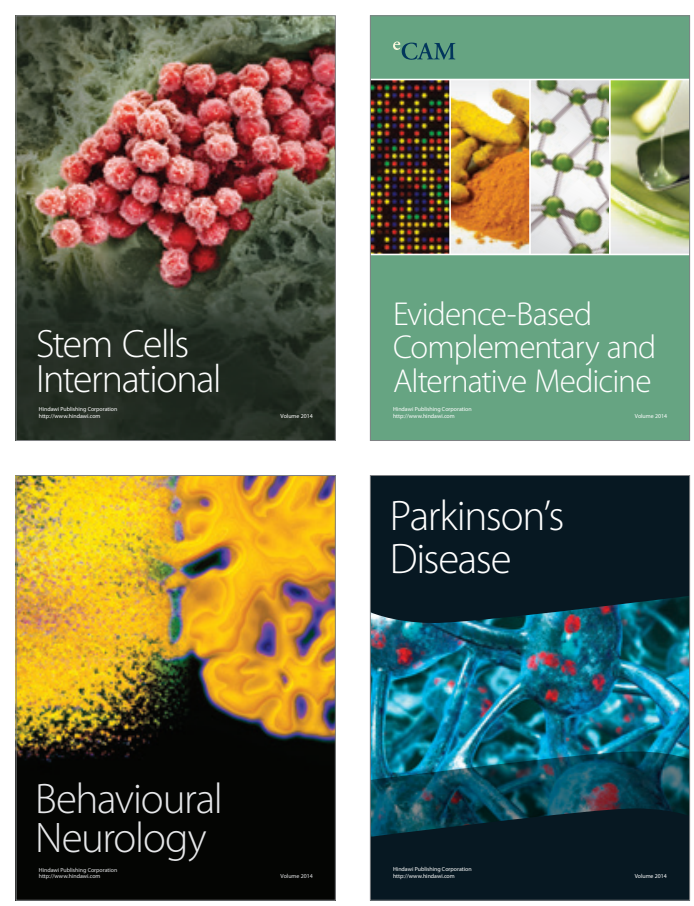

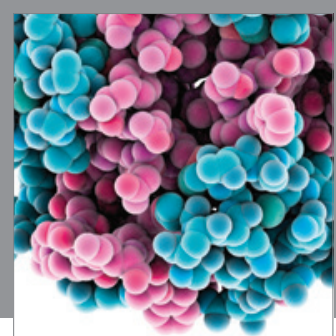

Journal of
Diabetes Research

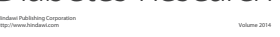

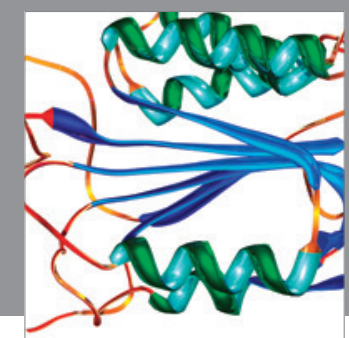

Disease Markers
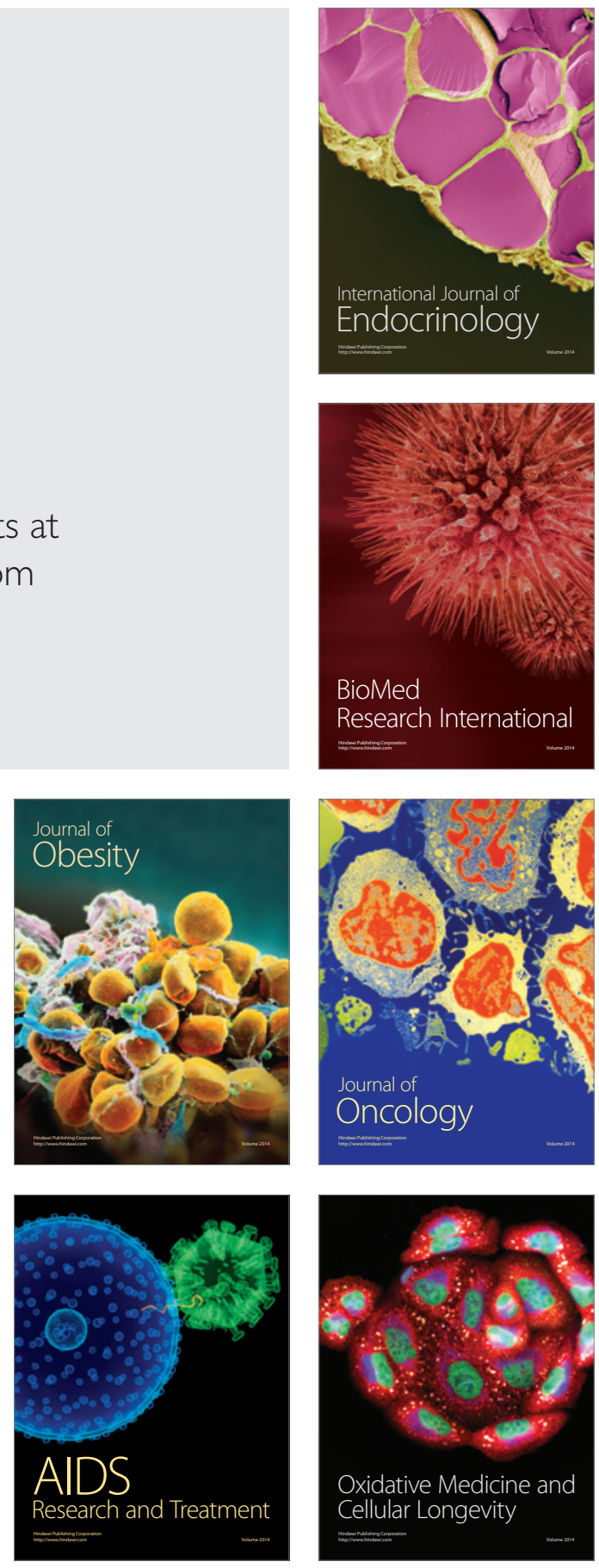